7th International Workshop on Astronomy and

Relativistic Astrophysics (IWARA 2016)

International Journal of Modern Physics: Conference Series

Vol. 45 (2017) 1760062 (4 pages)

(C) The Author(s)

DOI: $10.1142 / \mathrm{S} 201019451760062 \mathrm{X}$

\title{
Total, Elastic and Single Diffractive Cross Sections for LHC Energies
}

\author{
Mairon M. Machado \\ Instituto Federal Farroupilha (IFFAR) \\ Rua Otaviano Castilho Mendes, 355 \\ 97670-000 São Borja, Rio Grande do Sul, Brazil \\ mairon.machado@iffarroupilha.edu.br \\ Magno V. T. Machado \\ Instituto de Física \\ Universidade Federal do Rio Grande do Sul (UFRGS) \\ Av. Bento Gonçalves, 9500 \\ 91501-970 Porto Alegre, Rio Grande do Sul, Brazil \\ magnus@if.ufrgs.br
}

Published 15 August 2017

\begin{abstract}
In this contribution we provide predictions for total, elastic and single diffractive cross sections calculated for the proton-proton collisions at the LHC in centre-of-mass energies of $0.9,7,8$ and $14 \mathrm{TeV}$. We consider the framework of the Miettinen-Pumplin model which correctly describes the lower energy data available by Fermilab-Tevatron. Our predictions are based on the fitted parameters of the model for the Tevatron measurements and for TOTEM-LHC measurements at $7 \mathrm{TeV}$. We extrapolate the results for the higher energies runs of LHC and provide predictions for them. We verify that those prediction are in agreement with the recent CERN-ATLAS Collaboration result.
\end{abstract}

Keywords: Diffractive Cross Sections, Elastic Cross Sections, Miettinen-Pumplin Model.

PACS numbers: 13.60.Hb; 13.85.-t; 13.85.Lg; 13.85.Hd

\section{Introduction}

Since the earliest days of particle physics, measurements of the total proton-proton (proton-antiproton) cross sections and their theoretical understanding are topics of great interest. A number of approaches have been used to describe the existing measurements. Several models for soft interactions which were proposed prior to the measurements at the LHC are only marginally compatible with LHC data. Therefore, a consistent and simultaneous description of $\sigma_{t o t}, \sigma_{e l}$ and $\sigma_{d i f f}$ is a big challenge.

This is an Open Access article published by World Scientific Publishing Company. It is distributed under the terms of the Creative Commons Attribution 4.0 (CC-BY) License. Further distribution of this work is permitted, provided the original work is properly cited. 
The Miettinen-Pumplin model has described very well the Tevatron data for proton-antiproton collisions and the earlier CERN ISR data on proton-proton reactions ${ }^{1}$. Based on the validity of this model at Tevatron energy $(1.8 \mathrm{TeV})$, we are using its method to determine the total, elastic and diffractive cross sections at the LHC. The model ${ }^{2}$ is based on Good-Walker picture ${ }^{3}$ of soft difraction, where the state of the incidente hadron is expanded into a superposition of states wich are eingestates of diffraction. With a number of assumptions concerning parton distributions and interactions, the main formulae of Miettinen and Pumplin model for the differential cross sections depend on two parameters only. They are named $\beta$ and $G^{2}$, which are energy dependent. In terms of these parameter the differential cross sections for total, elastic and diffractive reactions are given by,

$$
\begin{aligned}
\frac{d \sigma_{t o t}}{d^{2} b} & =2\left[1-\exp \left(-G^{2} \frac{4}{9} e^{-b^{2} /(3 \beta)}\right)\right] ; \\
\frac{d \sigma_{e l}}{d^{2} b} & =\left[1-\exp \left(-G^{2} \frac{4}{9} e^{-b^{2} /(3 \beta)}\right)\right]^{2} ; \\
\frac{d \sigma_{S D}}{d^{2} b} & =\exp \left(-2 G^{2} \frac{4}{9} e^{-b^{2} /(3 \beta)}\right)\left[\exp \left(G^{2} \frac{1}{4} e^{-b^{2} /(2 \beta)}\right)-1\right] .
\end{aligned}
$$

The total cross sections $\sigma_{t o t}, \sigma_{e l}$ and $\sigma_{d i f f}$ are obtained after the integration over the impact parameter $b$. Only the single diffraction (SD) is considered in this model ${ }^{4}$.

The two free parameters $\beta$ and $G^{2}$ can be extracted for a given energy $\sqrt{s}$, using the experimental values for $\sigma_{t o t}$ and $\sigma_{e l}$. Thus, $\sigma_{S D}$ is obtained as a parameter free prediction. In Ref. ${ }^{1}$, predictions were made for low energies $(\sqrt{s}=30.4 \mathrm{GeV}$ and $\sqrt{s}=1800 \mathrm{GeV}$ ) (values appearing in the two first rows in Tab. 1). Now, considering the TOTEM Collaboration data from LHC (centre-of-mass energy of $7 \mathrm{TeV}$ ) (see Ref. ${ }^{5}$ ) we extract their values (see third row in Table 1). Using the IRS, Tevatron and TOTEM data, we perform a two-parameter fit (Fit 1 using the CDF data and

Table 1. Parameters of the Miettinen-Pumplin model, $\beta$ and $G^{2}$, extracted from total and elastic proton-antiproton (low energy) and proton-proton (LHC energy) cross sections in the energy

\begin{tabular}{|c|c|c|c|c|c|c|}
\hline $\begin{array}{l}\text { Data to fit } \\
\beta \text { and } G^{2}\end{array}$ & $\sqrt{s}[\mathrm{GeV}]$ & $\sigma_{t o t}[\mathrm{mb}]$ & $\sigma_{e l}[\mathrm{mb}]$ & $2 \sigma_{\text {diff }}[\mathrm{mb}]$ & $\beta\left[\mathrm{fm}^{2}\right]$ & $G^{2}$ \\
\hline ISR & 30.4 & $42.13 \pm 0.57$ & $7.17 \pm 0.34$ & $\longrightarrow$ & $0.284 \pm 0.018$ & $2.21 \pm 0.16$ \\
\hline $\mathrm{CDF}$ & 1800 & $80.03 \pm 2.24$ & $19.7 \pm 0.85$ & - & $0.337 \pm 0.030$ & $4.20 \pm 0.45$ \\
\hline TOTEM & 7000 & $98.30 \pm 0.24$ & $24.8 \pm 0.70$ & $0.201 \pm 0.03$ & $0.400 \pm 0.052$ & $4.42 \pm 0.56$ \\
\hline Fit 1 & 8000 & 92.34 & 23.91 & 9.23 & 0.36 & 4.67 \\
\hline Fit 2 & 8000 & 88.11 & 22.25 & 9.28 & 0.36 & 4.43 \\
\hline Fit 1 & 13000 & 98.46 & 26.28 & 1.46 & 0.37 & 5.02 \\
\hline Fit 2 & 13000 & 93.73 & 24.39 & 1.47 & 0.37 & 4.73 \\
\hline Fit 1 & 14000 & 99.32 & 26.63 & 1.46 & 0.37 & 5.07 \\
\hline Fit 2 & 14000 & 94.64 & 24.74 & 1.47 & 0.37 & 4.78 \\
\hline
\end{tabular}
range $\sqrt{s}=30-7000 \mathrm{GeV}$. Predictions for these cross sections, including $\sigma_{S D}$ for LHC energies (8, 13 and $14 \mathrm{TeV})$. See text for discussions. 


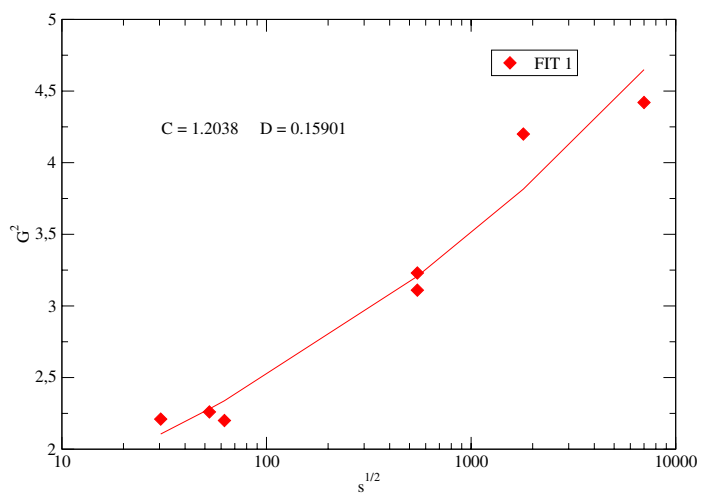

Fig. 1. $G^{2}$ predictions in terms of $\sqrt{s}$.

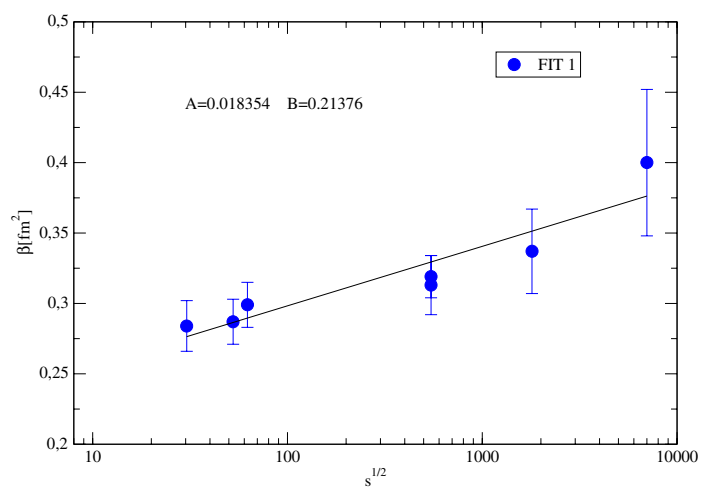

Fig. 2. $\beta$ predictions in terms of $\sqrt{s}$.

Fit 2 using E811 data) for the energy dependence of the parameters, as presented in Figs. 1 and 2 (Fit 1). Finally, we extrapolate the values of $\beta$ and $G^{2}$ to higher energies at LHC and perform predictions for total, elastic and single diffraction cross sections to $\sqrt{s}=8,13$ and $14 \mathrm{TeV}$ (the values are shown in Tab. 1).

\section{Results}

Our predictions are made using the recent data of $\sigma_{t o t}$ and $\sigma_{e l}$ from TOTEM Collaboration $(\sqrt{s}=7 \mathrm{TeV})$ (see Ref. $\left.{ }^{5}\right)$. The predictions in Ref. ${ }^{1}$ just included data from Tevatron, and in this way, our predictions are more updated. They are in very good agreement with recent data extracted from ATLAS $(\sqrt{s}=8 \mathrm{TeV})$ (see Ref. $\left.{ }^{7}\right)$, which gives $\sigma_{\text {tot }}=96.07 \pm 0.92 \mathrm{mb}$ and $\sigma_{e l}=24.33 \pm 0.39 \mathrm{mb}$ (errors summed into quadrature).

Figures 1 and 2 show Fit 1 for $G^{2}$ and $\beta$ parameters, respectively, in terms of energy $(\sqrt{s})$. The predicted values for cross sections at $\sqrt{s}=14 \mathrm{TeV}$ are in good agreement with previous results presented in Ref. ${ }^{1}$, which are $\sigma_{\text {tot }}=86.4$ 
$\mathrm{mb}, \sigma_{e l}=21.2 \mathrm{mb}$ and $\sigma_{d i f f}=9.5 \mathrm{mb}$. In those figures, $C$ and $D$ are parameters provided by a power regression between the data (in the form $y=C * D^{B}$ ), while $A$ and $B$ are parameters provided by a logarithmic regression between data (in the form $y=A+B * \ln (x))$.

\section{Conclusion}

The total, elastic and single diffractive cross sections are predicted for the LHC energies using the Miettinen-Pumplin mode. We have found the total inclusive cross section to be $\sigma_{t o t}=92.34 \mathrm{mb} ; 98.46 \mathrm{mb}$ and $99.32 \mathrm{mb}$ for $\sqrt{s}=8 ; 13$ and $14 \mathrm{TeV}$, respectively (Fit 1). Considering Fit 2, one obtains $\sigma_{\text {tot }}=88.11 \mathrm{mb}, 93.73 \mathrm{mb}$ and $94.64 \mathrm{mb}$ for the same energies. The uncertainty related to the choice of distinct Fits(1 or 2) is around $5 \%$.

Our predictions for $8 \mathrm{TeV}$ is in good agreement to the recent data measured by ATLAS Collaboration. Our results are also compatible with more involved models for soft interactions, for instance those presented in Ref. ${ }^{6}$.

As a short conclusion, it was shown that the Miettinen and Pumplin model is a good framework to describe diffraction dissociation in hadron-hadron collisions considering energies in the terascale regime.

\section{Acknowledgments}

This work was supported by CNPq and FAPERGS, Brazil. M. M. Machado would like to thank the Instituto Federal Farroupilha for financial support.

\section{References}

1. S. Sapeta, Phys. Lett. B 597, 352 (2004).

2. H. I. Miettinen and J. Pumplin, Phys. Rev. D 18, 1696 (1978).

3. M. L. Good and W. D. Walker, Phys. Rev. 120, 1857 (1960).

4. ALICE Collaboration (M. G. Poghosyan et al.), Journal of Physics G: Nuclear and Particle Physics 38, 12 (2011).

5. TOTEM Collaboration (G. Antchev et al.), Europhys. Lett. 96, 21002 (2011).

6. E. Gotsman, E. Levin and U. Maor, Phys. Rev. D 85, 094007 (2012).

7. ATLAS Collaboration (G. Aad et al.), Phys. Lett. B 761, 158 (2016). 